\title{
SURFACES IN CONSTANT CURVATURE MANIFOLDS WITH PARALLEL MEAN CURVATURE VECTOR FIELD
}

\author{
BY DAVID A. HOFFMAN \\ Communicated by James Serrin, July 8, 1971
}

I. Statement of results. For an (n)-dimensional Riemannian manifold $M^{n}$, isometrically immersed in an $(n+k)$-dimensional Riemannian manifold $M_{(c)}^{(n+k)}$ of constant sectional curvature $c$, let $H$ denote the mean curvature vector field of $M^{n} . H$ is a section of the normal bundle $N M^{n}$ of the immersion. When $n=2, k=1$, and $c=0$ (a surface immersed in $E^{3}$ ), the requirement $|H|=$ constant is classical constant mean curvature. If $k>1$, however, the condition $|H|=$ constant is usually too weak to prove reasonable generalizations of the classical theorems for surfaces of constant mean curvature in $E^{3}$. We investigate a stronger requirement on $H$; namely, that $H$ be parallel with respect to the induced connection in the normal bundle (for definitions, see II). Then using an analytic construction first employed by H. Hopf [2], we obtain

THEOREM 1. A compact surface $M^{2}$ of genus 0 immersed in $M^{4}(c), c \geqq 0$, upon which $H$ is parallel in the normal bundle, is a sphere of radius $1 /|H|$.

This generalizes a theorem of Hopf, who proved that the only immersed surfaces in $E^{\mathbf{3}}$ of genus 0 with constant mean curvature are spheres [2, Chapter 7, §4]. For complete surfaces in $E^{4}$, we prove

THEOREM 2. A complete surface $M^{2}$, immersed in $E^{4}$, whose Gauss curvature does not change sign, and for which $H$ is parallel in the normal bundle $N^{2}$, is a minimal surface $(H \equiv 0)$, a sphere, a right circular cylinder, or a product of circles $S^{1}(r) \times S^{1}(\rho)$, where $|H|=\frac{1}{2}\left(1 / r^{2}+1 / \rho^{2}\right)^{1 / 2}$.

This extends a theorem of Klotz and Osserman for complete surfaces of constant scalar mean curvature in $E^{3}[5]$. It can also be generalized to immersions into $\bar{M}_{(c)}^{4}, c \geqq 0$. Theorem 2 is proved in two steps. First we prove

THEOREM 3. A piece of immersed surface $M^{2}$ in $E^{4}$, satisfying the conditions of Theorem 2 with $H \neq 0$, is either a sphere or it is flat $(K=0)$.

Then we establish the following characterization of flat surfaces in $E^{4}$ with parallel mean curvature vector fields:

AMS 1970 subject classifications. Primary 53C40, 53A05, 53C45, 53B20; Secondary 53A10, 30A52.

Key words and phrases. Constant curvature manifolds, parallel mean curvature vector, constant mean curvature, complete surfaces, Codazzi equations, conformal coordinates, pseudo-umbilical, parallel normal bundle. 
THEOREM 4. A piece of immersed surface $M^{2}$ in $E^{4}$ with parallel mean curvature vector $H \neq 0$ and $K \equiv 0$ is a piece of $S^{1}(r) \times S^{1}(\rho)$ : the product of two circles of radius $r$ and $\rho$ with the standard flat immersion. ( $\rho$ may be infinite, in which case we have a right circular cylinder.)

Theorem 2 generalizes to immersions in $S^{4}(c)$.

Surfaces in $E^{n}$ which lie minimally in hyperspheres of radius $r$ have the same mean curvature vectors as the hypersphere, and consequently have parallel mean curvature vector fields. Such surfaces are pseudo-umbilic $\left(\varphi_{3} \equiv 0\right.$ in the lemma in II). In this case, Itoh [3] has proven a special case of Theorem 2 for immersions in $E^{4}$ (see also Chen, [1]). For minimal surfaces in $S^{4}$, Ruh [8] has proven a case of Theorem 1, using methods similar to the basic lemma in II. For a wide variety of examples of complete minimal surfaces in $S^{3}$, see Lawson [6].

It is possible to show the existence of surfaces in $E^{n}$ and $S^{n}(c)$ with parallel $H$ and $\varphi_{3} \neq 0$ (i.e. they do not lie minimally in hyperspheres). The method employed uses a theorem due to Szczarba [9] on existence of immersions in constant curvature manifolds with codimension $k>1$.

II. Definitions and Main Lemma. $\bar{\nabla}$ denotes covariant differentiation on $\bar{M}_{(c)}^{n+k}$, and $\nabla$ denotes covariant differentiation on $M^{n} \subset \bar{M}^{n+k}$. For $X, Y$, sections of $T M^{n}, \nabla_{X} Y=\left[\bar{\nabla}_{X} Y\right]^{T}$ where []$^{T}$ is projection onto $T M^{n}$. []$^{N}$ is projection onto $N M^{n}$.

Definitions. $B(X, Y)=\left[\bar{\nabla}_{X} Y\right]^{N} . B$ is the second fundamental form tensor of the immersion. Similarly for $N$, a section of $N M^{n}, D_{X} N=\left[\bar{\nabla}_{X} N\right]^{N}$. $D$ defines a connection on $N M^{n} . A(X, N)=\left[\bar{\nabla}_{X} N\right]^{T} . A$ is a tensor: $A_{p}$ : $T M^{n} \times N M^{n} \rightarrow T M^{n}$ is bilinear.

For an orthonormal framing $\left(e_{1} \cdots e_{n}\right)$ of $T M^{n}, H=(1 / n) \sum_{i=1}^{n} B\left(e_{i}, e_{i}\right)$. This definition of $H$ is independent of the framing. A normal vector field $N$ is said to be parallel in the normal bundle $N M^{n}$ if $D_{X} N=0$ for all $X$ in $T M^{n}$. This condition implies $|N|=$ const. Thus our assumption that $H$ is parallel in $N M^{n}$ includes constant mean curvature. $(|H|=c$.)

The Gauss and Codazzi equations, for $X, Y, Z$ sections in $T M^{n}$, are

(1) $R(X, Y) Z=c\{\langle X, Z\rangle Y-\langle Y, Z\rangle X\}+A(X, B(Y, Z))-A(Y, B(X, Z)$ ),

$$
\left(\nabla_{X} B\right)(Y, Z)=\left(\nabla_{Y} B\right)(X, Z)
$$

where $\left(\nabla_{X} B\right)(Y, Z)=D_{X}(B(Y, Z))-B\left(\nabla_{X} Y, Z\right)-B\left(Y, \nabla_{X} Z\right)$ (for a reference for the above definitions and equations, see [4, Chapter 7]).

For $X, Y$ in $T M^{n}, N$ in $N M^{n}, \tilde{R}(X, Y) N=D_{X} D_{Y} N-D_{Y} D_{X} N-D_{[X Y]} N$ is the curvature tensor for $D$. For $\tilde{R}$, there is a Gauss-type equation

$$
\tilde{R}(X, Y) N=B(X, A(N, Y))-B(Y, A(N, X))
$$

and an equation, analogous to (2),

$$
\left(\nabla_{X} A\right)(Y, N)=\left(\nabla_{Y} A\right)(X, N) .
$$


For a unit normal vector $e_{\alpha}$ at $p \in M^{n}$, the matrix $\left(\lambda_{i j}^{\alpha}\right)=\left(B\left(e_{i}, e_{j}\right) \cdot e_{\alpha}\right)$ is the "second fundamental form matrix in the $\alpha$ direction." We specify $H /|H|$ as $e_{n+1}$ when $H \neq 0$. Considering an immersed surface $(n=2)$ given in conformal coordinates $(u, v): d s^{2}=E\left(d u^{2}+d v^{2}\right)$, we associate to it a natural framing

$$
\left(e_{1}, e_{2}\right)=\left(\frac{\partial}{\partial u} / \sqrt{ } E, \frac{\partial}{\partial v} / \sqrt{ } E\right)
$$

of the tangent bundle, $T M^{2}$.

Lemma. For an immersed surface, $M^{2} \hookrightarrow \bar{M}_{(c)}^{n+k}$ in conformal coordinates, let $H \neq 0$ and $e_{\alpha}$ be a unit normal vector field with $e_{\alpha} \cdot H=0$ :

(a) if $H$ is parallel in $N M^{2}$, then $\varphi_{3}=E\left\{\frac{1}{2}\left(\lambda_{11}^{3}-\lambda_{22}^{3}\right)-i \lambda_{12}^{3}\right\}$ is an analytic function of $z=u+i v$;

(b) if $e_{\alpha}$ is parallel in $N M^{2}$, then $\varphi_{\alpha}=E\left\{\lambda_{11}^{\alpha}-i \lambda_{12}^{\alpha}\right\}$ is an analytic function of $z$;

(c) if $k=2$ and $H$ is parallel, then $e_{\alpha}$ is parallel, and both $\varphi_{3}$ and $\varphi_{\alpha}$ are analytic;

(d) under the conditions of (a) and (b), either $\varphi_{3} \equiv 0$ or $\varphi_{\alpha}=\kappa \varphi_{3}$ where $\kappa$ is a real constant.

SKETCH OF PROOF. (a) Using equation (4) with $X=\partial / \partial u, Y=\partial / \partial v$, $N=H$, and the assumption that $H$ is parallel, the equations

(5) $\left(E \lambda_{11}^{3}\right)_{v}-\left(E \lambda_{12}^{3}\right)_{u}=\frac{1}{2} E_{v}\left(\lambda_{11}^{3}+\lambda_{22}^{3}\right), \quad\left(E \lambda_{12}^{3}\right)_{u}-\left(E \lambda_{22}^{3}\right)_{v}=\frac{1}{2} E_{u}\left(\lambda_{11}^{3}+\lambda_{22}^{3}\right)$

are obtained. (5) is in the same form as the Codazzi equations in conformal coordinates for surfaces in $E^{3}$, only it is expressed for the distinguished normal $e_{3}=H /|H|$. Since $\lambda_{11}^{3}+\lambda_{22}^{3}=2|H|=$ constant, (5) reduces to the Cauchy-Riemann equations for $\varphi_{3}$.

(b) Proof follows that of (a), using the fact that $\lambda_{11}^{\alpha}+\lambda_{22}^{\alpha}=0$.

(c) Since $N M^{2}$ is 2-dimensional, the assumption that $H$ is parallel forces $e_{\alpha}$ to be parallel. Then (a) and (b) imply analyticity.

(d) Using equation (3) with

$$
X=\frac{\partial}{\partial u_{1}} / \sqrt{ } E, \quad Y=\frac{\partial}{\partial u_{2}} / \sqrt{ } E, \quad \text { and } \quad N=e_{3},
$$

we obtain, using the fact that $e_{3}$ is parallel,

$$
0=\left(\sum_{k=1}^{2} \lambda_{k 2}^{3} \lambda_{k 1}^{\alpha}-\lambda_{k 1}^{3} \lambda_{k 2}^{\alpha}\right)
$$

Note that (6) implies $\operatorname{Im}\left(\varphi_{\alpha} \cdot \bar{\varphi}_{3}\right)=0$. So if $\varphi_{3} \not \equiv 0, \varphi_{\alpha} / \varphi_{3}=\varphi_{\alpha} \cdot \bar{\varphi}_{3} /\left|\varphi_{3}\right|^{2}$ is real. By (a) and (b), it is also meromorphic, hence constant.

III. Proof of Theorems (Sketch). Theorem 1 is proved by constructing an analytic differential $\theta_{3}$ out of the functions $\varphi_{3}(z)$ of the lemma: in local coordinates, $\theta_{3}=\varphi_{3} d z^{2}$. Since $M^{2}$ is of genus $0, \theta_{3}$ must be identically zero. 
Hence $\varphi_{3}(z) \equiv 0$. This implies that $M^{2}$ is pseudo-umbilic $\left(\lambda_{11}^{3}=\lambda_{22}^{3}\right.$, $\lambda_{12}^{3}=0$ ). The function $\varphi_{4}$ associated with $e_{4}, e_{4} \cdot H=0$ is also zero by a similar argument. Hence $M^{2}$ is totally umbilic. This implies that $M^{2}$ is immersed as a sphere.

To prove Theorem 3, we can consider on $M^{2}$ the quadratic analytic differentials $\theta_{3}$ and $\theta_{4}$ given locally by $\varphi_{3} d z^{2}$ and $\varphi_{4} d z^{2}$ (where $\varphi_{3}, \varphi_{4}$, and $z$ are as in the lemma). If $K \geqq 0, M^{2}$ is either compact or parabolic by a theorem of Huber (see [5, p. 316]). If it is compact, then either $K \equiv 0$ or $M^{2}$ is of genus 0 . The genus 0 case is a sphere by Theorem 1 .

If $K \leqq 0$, then $|H|^{2}-K>|H|^{2}>0$. In a neighborhood of each point, we introduce the new metric $d \tilde{s}^{2}=E\left(|H|^{2}-K\right)^{1 / 2}\left(d u^{2}+d v^{2}\right)$. Using the equality

$$
\left|\varphi_{3}\right|^{2}+\left|\varphi_{4}\right|^{2}=E^{2}\left(|H|^{2}-K\right)
$$

and part (d) of the lemma to show that $\Delta \log \left(\left|\varphi_{3}\right|^{2}+\left|\varphi_{4}\right|^{2}\right)=0$, we establish that $d \tilde{s}^{2}$ is a flat metric. Therefore, the univeral covering surface $\tilde{M}^{2}$ of $M^{2}$ is conformally the plane. The function $|H|^{2}-K$ is easily seen to be superharmonic. Since it is bounded below, it must be constant. Hence $K$ is constant, and must be zero.

Theorem 4 is proved by introducing conformal coordinates $(u, v)$ such that $E \equiv 1$. The lemma is used to show that all $\lambda_{i j}^{\alpha}$ are constant. Then a rotation of coordinates puts the immersion into the form

$$
(u, v) \rightarrow\left(r \cos \frac{u}{r}, r \sin \frac{u}{r}, \rho \cos \frac{v}{\rho}, \rho \sin \frac{v}{\rho}\right) .
$$

The constants $r$ and $\rho$ are determined from the $\lambda_{i j}^{\alpha}$ and $|H|$. This immersion is the standard flat immersion of the plane into $E^{4}$ as a product of circles.

\section{BIBLIOGRAPHY}

1. a) B.-Y. Chen, Minimal surfaces in $S^{m}$ with Gauss curvature $\leqq 0$, Proc. Amer. Math. Soc. (to appear).

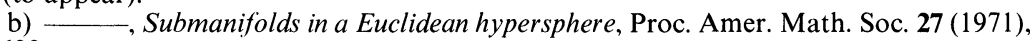
627-629.

2. H. Hopf, Lectures on differential geometry in the large, Stanford University, Stanford, Calif., 1956 (mimeographed notes).

3. T. Itoh, Complete surfaces in $E^{4}$ with constant mean curvature, Kōdai Math. Sem. Rep. 22 (1970), 150-158.

4. S. Kobayashi and K. Nomizu, Foundations of differential geometry. Vol. II, Interscience Tracts in Pure and Appl. Math., no. 15, Interscience, New York, 1969. MR 38 \# 6501.

5. T. Klotz and R. Osserman, Complete surfaces in $E^{3}$ with constant mean curvature, Comment. Math. Helv. 41 (1966/67), 313-318. MR 35 \#2213.

6. H. B. Lawson, Jr., Complete minimal surfaces in $S^{3}$, Ann. of Math. (2) 92 (1970), 335-374.

7. T. Ôtsuki, A theory of Riemannian submanifolds, Kōdai Math. Sem. Rep. 20 (1968), 282-295. MR 38 \#2707.

8. E. A. Ruh, Minimal immersions of 2-spheres in $S^{4}$, Proc. Amer. Math. Soc. 28 (1971), 219.

9. R. H. Szczarba, On existence and rigidity of isometric immersions, Bull. Amer. Math. Soc. 75 (1969), 783-787; Addendum, ibid. 76 (1970), 425. MR 40 \# 3471.

Department of Mathematics, Stanford University, Stanford, California 94305 Current address: Department of Mathematics, University of Durham, Durham, ENGLAND 